\title{
A topological insulator surface under strong Coulomb, magnetic and disorder perturbations
}

\author{
L. Andrew Wray ${ }^{1,2,3}$, Su-Yang Xu' ${ }^{1}$, Yuqi Xia', David Hsieh ${ }^{1}$, Alexei V. Fedorov ${ }^{3}$, Yew San Hor ${ }^{4}$, \\ Robert J. Cava ${ }^{4}$, Arun Bansil ${ }^{5}$, Hsin Lin ${ }^{5}$ and M. Zahid Hasan ${ }^{1,2,6 \star}$
}

\begin{abstract}
Topological insulators embody a state of bulk matter characterized by spin-momentum-locked surface states that span the bulk bandgap ${ }^{1-7}$. This highly unusual surface spin environment provides a rich ground for uncovering new phenomena ${ }^{4-24}$. Understanding the response of a topological surface to strong Coulomb perturbations represents a frontier in discovering the interacting and emergent many-body physics of topological surfaces. Here we present the first controlled study of topological insulator surfaces under Coulomb and magnetic perturbations. We have used time-resolved deposition of iron, with a large Coulomb charge and significant magnetic moment, to systematically modify the topological spin structure of the $\mathrm{Bi}_{2} \mathrm{Se}_{3}$ surface. We observe that such perturbation leads to the creation of odd multiples of Dirac fermions and that magnetic interactions break time-reversal symmetry in the presence of band hybridizations. We present a theoretical model to account for the observed electron dynamics of the topological surface. Taken collectively, these results are a critical guide in controlling electron mobility and quantum behaviour of topological surfaces, not only for device applications but also in setting the stage for creating exotic particles such as axions or imaging monopoles on the surface.
\end{abstract}

Bismuth selenide has been experimentally discovered by angleresolved photoemission spectroscopy (ARPES) to be a topological insulator with a large bulk bandgap ( $2300 \mathrm{meV}$; refs 6,7$)$. Spinresolved photoemission studies reveal that surface electrons in $\mathrm{Bi}_{2} \mathrm{Se}_{3}$ form a Dirac cone spanning the bulk insulating gap, composed of spin-momentum-locked helical states (Fig. 1a). The Fermi level of grown crystals is usually found to be located in the bulk conduction band due to selenium vacancy defects; however, it was subsequently shown that with $\mathrm{Ca}$ doping or $\mathrm{NO}_{2}$ surface deposition the Fermi level can be placed at the Dirac point, reaching the topological transport regime ${ }^{6}$, and magnetic interactions can be controlled through magnetic ions such as Fe or Mn (refs 9, 10). Placing the Fermi level at the Dirac point in the presence of magnetic impurities in the bulk can lead to a small gap at the Fermi level (Fig. 1b); however, the full character of this gap cannot be decisively resolved owing to the lineshape broadening effects. So far, no systematic surface deposition of magnetic impurities to elicit a large systematic magnetic response and bring about controlled changes in the surface band structure has been explored. Probing the effect of magnetic perturbation on the surface is more relevant for potential applications than the previous studies of bulk magnetic dopants, because topological insulators need to be in contact with large-moment ferromagnets and superconductors for device applications ${ }^{11-17}$. The focus of this letter is the exploration of topological insulator surface electron dynamics in the presence of magnetic, charge and disorder perturbations from deposited iron on the surface.

Bismuth selenide cleaves on the (111) selenium surface plane, providing a homogeneous environment for deposited atoms. Iron deposited on a $\mathrm{Se}^{2-}$ surface is expected to form a mild chemical bond, occupying a large ionization state between $2^{+}$and $3^{+}$ with roughly $4 \mu_{\mathrm{B}}$ magnetic moment ${ }^{25}$. As seen in Fig. 1d the surface electronic structure after heavy iron deposition is greatly altered, showing that a significant change in the surface electronic environment has been achieved. Five (odd number of) surface bands intersect the Fermi level rather than just one, and extend below the Fermi level in the form of multiple Dirac cones. Unlike the bulk electronic states of $\mathrm{Bi}_{2} \mathrm{Se}_{3}$ (ref. 22), these features have no $z$-axis momentum dependence, confirming that they are largely two dimensional in nature (Fig. 1e).

Figure 2 shows the surface evolution as a function of deposition time in crystals with chemical compositions of (sample no 1) $\mathrm{Bi}_{1.9975} \mathrm{Ca}_{0.0025} \mathrm{Se}_{3}$ and (sample no 2) $\mathrm{Bi}_{2} \mathrm{Se}_{3}$ varied to tune the carrier density. The rate of iron deposition was similar to $4 \%$ of a monolayer per minute, as detailed in Supplementary Information, and deposition data for an extra sample with intermediate carrier concentration (sample no 3) are also presented in the Supplementary Information. The bulk electronic state of sample no 1 before deposition is slightly $\mathrm{p}$ type with significant resistivity $(\rho=23 \mathrm{~m} \Omega \mathrm{cm})$, and sample no 2 is electron doped from Se vacancy defects in as-grown $\mathrm{Bi}_{2} \mathrm{Se}_{3}$. In each case, it is observed that the presence of positively charged Fe surface ions progressively lowers the energy of the surface state and causes the appearance of new surface states with energy-momentum contours similar to the bottom of the bulk conduction band.

The changes brought on by iron deposition can be seen most strikingly in measurements on the bulk p-type sample no 1 . After approximately six minutes of deposition, new surface states that do not exist in unperturbed samples become visible at the Fermi level, with new Dirac points labelled D1 and D2 above the Dirac cone that is seen in as-grown $\mathrm{Bi}_{2} \mathrm{Se}_{3}$ (D0). It is also after approximately six minutes of deposition that a gap begins to be apparent at the D0 Dirac node, shown by a parabolic shape near the Dirac node, separating the upper and lower Dirac cones of the original surface state. This gap can be seen clearly at different incident photon energies in Fig. 2d, confirming that it is a feature of the surface and

\footnotetext{
'Department of Physics, Joseph Henry Laboratories, Princeton University, Princeton, New Jersey 08544, USA, 2 Princeton Center for Complex Materials, Princeton University, Princeton, New Jersey 08544, USA, ${ }^{3}$ Advanced Light Source, Lawrence Berkeley National Laboratory, Berkeley, California 94305 , USA, ${ }^{4}$ Department of Chemistry, Princeton University, Princeton, New Jersey 08544, USA, ${ }^{5}$ Department of Physics, Northeastern University, Boston, Massachusetts 02115, USA, ${ }^{6}$ Princeton Institute for Science and Technology of Materials, Princeton University, Princeton, New Jersey 08544, USA. *e-mail:mzhasan@Princeton.edu.
} 
a



b
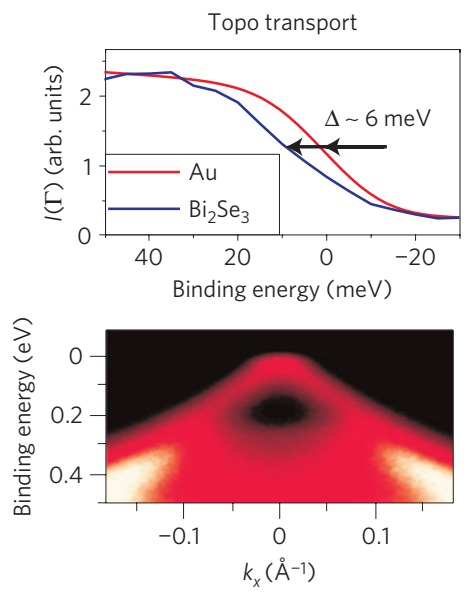

c

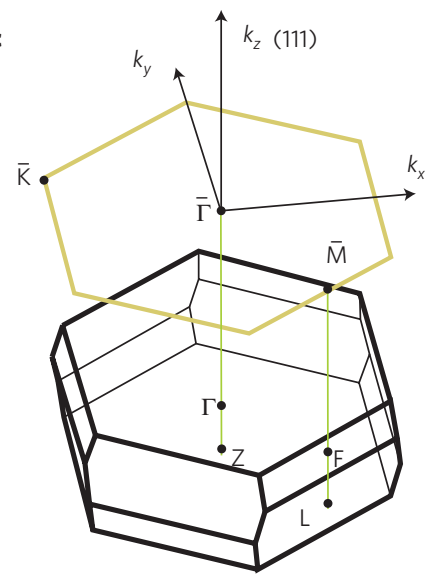

d

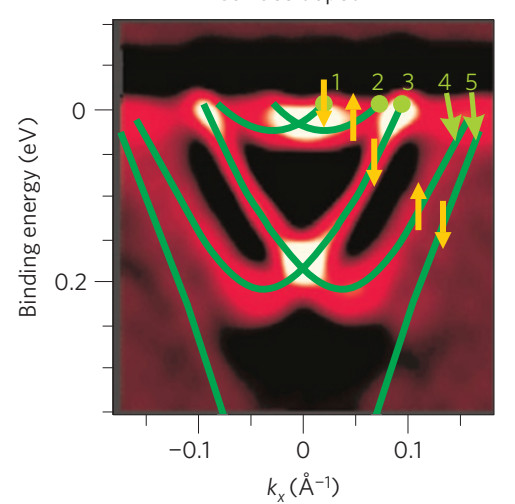

e



$33 \mathrm{e}$

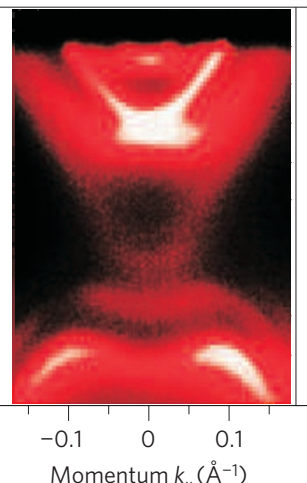

$29 \mathrm{eV}(Z)$

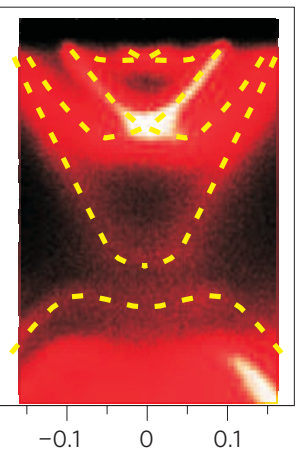

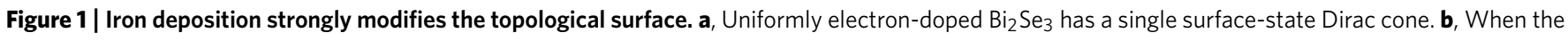
surface chemical potential of as-grown $\mathrm{Bi}_{2} \mathrm{Se}_{3}$ is lowered to the Dirac point by $\mathrm{NO}_{2}$ deposition, we observe a slight gap in the leading edge of $\mathrm{ARPES}$ intensity. c, The hexagonal surface Brillouin zone of $\mathrm{Bi}_{2} \mathrm{Se}_{3}$ is drawn above a diagram of the three-dimensional bulk Brillouin zone. $\mathbf{d}$, A second-derivative image of new surface states in $\mathrm{Bi}_{2} \mathrm{Se}_{3}$ (sample no 1) after surface iron deposition is labelled with numerically predicted spin texture from Fig. 36 . e, Low-energy features from $\mathbf{d}$ have no $z$-axis momentum dispersion, as seen from the data taken with varying incident photon energy (37-29 eV), confirming the two-dimensional character of the state.

not the bulk electronic band structure. Electron velocities (band slope) near the D1 and D2 Dirac points increase monotonically as iron is added, showing that iron is increasing the 'Rashba' interaction term $((\mathbf{k} \times \hat{z}) \cdot \boldsymbol{\sigma}$, with $\boldsymbol{\sigma}$ representing the Pauli matrices $)$ identified in theoretical models ${ }^{3}$. The number of surface bands intersecting the Fermi level between the $\bar{\Gamma}$ and $\bar{M}$ points progresses from one to three to five, with one band contributed by the original (D0) Dirac cone and two more bands contributed by each of the new (D1, D2) Dirac points. This is consistent with the $\operatorname{Mod}(2)$ character of surface electrons on a crystal with bulk topological insulator order, that the topological surface likes to maintain an odd number of Dirac states. After 12 min of deposition, the binding energy of the D0 Dirac point was found to have sunk by approximately $0.6 \mathrm{eV}$ in energy, and the electron binding energies ceased to change under further deposition. When the chemical potential is positioned above the bulk conduction-band minimum, as in sample no 2 (Fig. 2c), the dispersion of new surface states across the full bulk bandgap is visible within the photoemission image. A new, strongly split surface band is observed in sample no 2 after five minutes of Fe deposition with a (D1) Dirac point at the $\bar{\Gamma}$ point, but no further (for example, D2) bands appeared after longer deposition.

Theoretical simulation of non-magnetic surface Coulomb perturbation on $\mathrm{Bi}_{2} \mathrm{Se}_{3}$ is shown in Fig. $3 \mathrm{a}$, and qualitatively reproduces the progressive appearance of new Dirac points with increasing iron deposition. Through comparison with our numerical result, we can see that the experimentally observed surface states begin to pair off at momentum separation greater than $\sim 0.1 \AA^{-1}$ from the Brillouin zone centre, with the upper D0 Dirac cone approaching degeneracy with the lower D1 band, and the upper D1 band connecting to the lower D2 band. The partner-swapping connectivity observed in the simulation and data is a simple way by which new states can be added to the surface band structure without disrupting the surface conditions required by the bulk topological insulator order of $\mathrm{Bi}_{2} \mathrm{Se}_{3}$ (ref. 3). The spin-splitting of topological surface bands is often discussed as a special case of the Rashba effect (see, for example, refs 3,20), in which surface electronic states become spin-split by an energy proportional to their momentum $\mathbf{k}$. Our data and simulations show that this description is only accurate for $\mathrm{Bi}_{2} \mathrm{Se}_{3}$ in a small part of the Brillouin zone surrounding the Brillouin zone centre, because at momenta further from the $\bar{\Gamma}$ point the electronic states pair off and are nearly spin degenerate (see Fig. 3b). This can be understood because the origin of the topological insulator state in $\mathrm{Bi}_{2} \mathrm{Se}_{3}$ is a symmetry inversion that occurs at the $\Gamma$ point ${ }^{7,18}$, and the electronic states close to the Brillouin-zone boundary are similar to those of topologically trivial materials. The role of magnetic domains in reshaping low-energy band structure is expected to be more subtle than Coulomb perturbations, and may be limited to changes near the Dirac points and in self-energy lineshape effects (see Fig. 3c,d).

Recent theoretical studies suggest that the physical environment of magnetic impurities on a topological surface is very different 

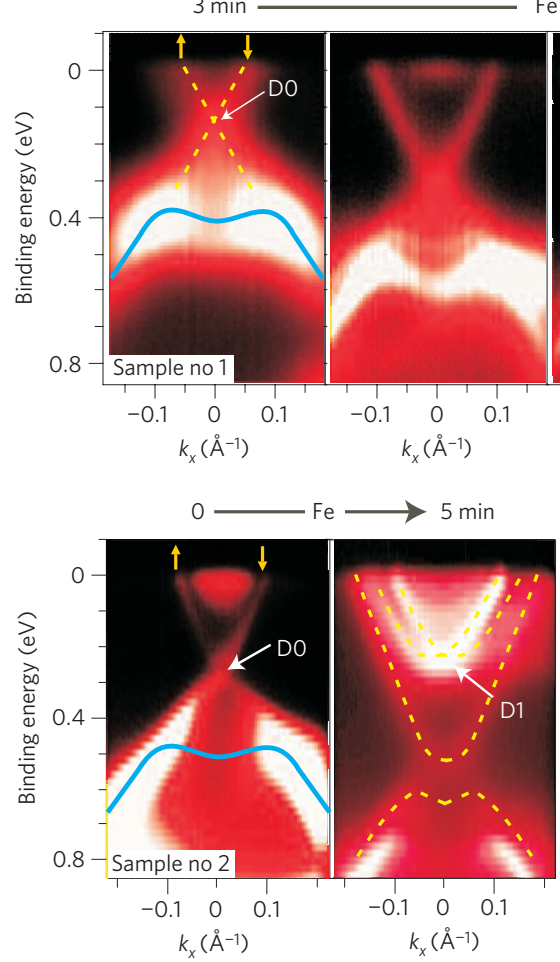

Fe deposition time



$12 \mathrm{~min}$



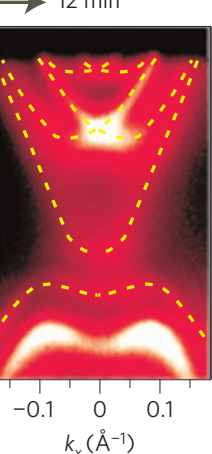

b

d

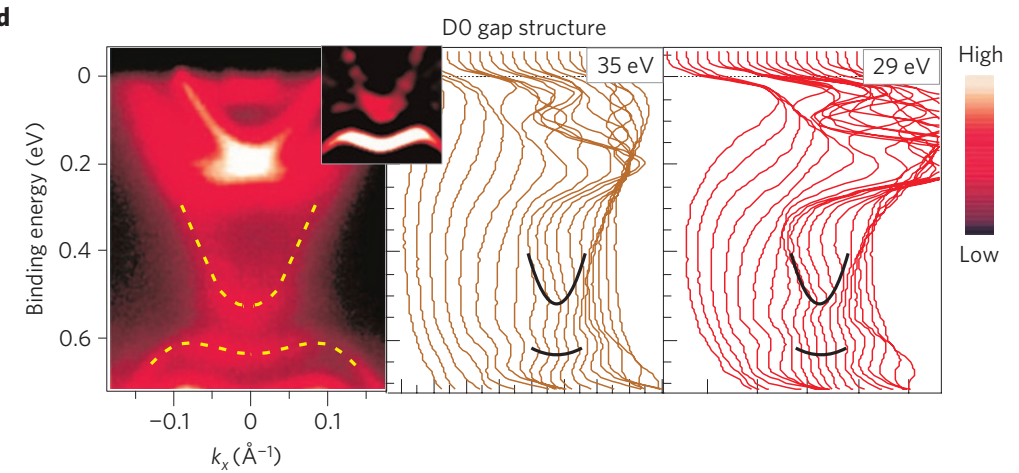

Figure 2 | Iron doping creates five helical Dirac cones. a, Progressive surface doping of optimally insulating $\mathrm{Ca}_{x} \mathrm{Bi}_{2-x} \mathrm{Se}_{3}$ (sample no 1) causes the successive appearance of two new Dirac points ('D1' and 'D2'). b. Second-derivative images of the Fermi surface and low-energy surface states for Fe-deposited sample no 1. c, Fe deposition on as-grown n-type $\mathrm{Bi}_{2} \mathrm{Se}_{3}$ (sample no 2) results in a similar final spectrum to a, but with only a single new Dirac point. d, The iron-induced DO gap is observed at different binding energies for sample no 1 and (inset) in a second-derivative image. The existence of the surface bandgap is not sample dependent.

a

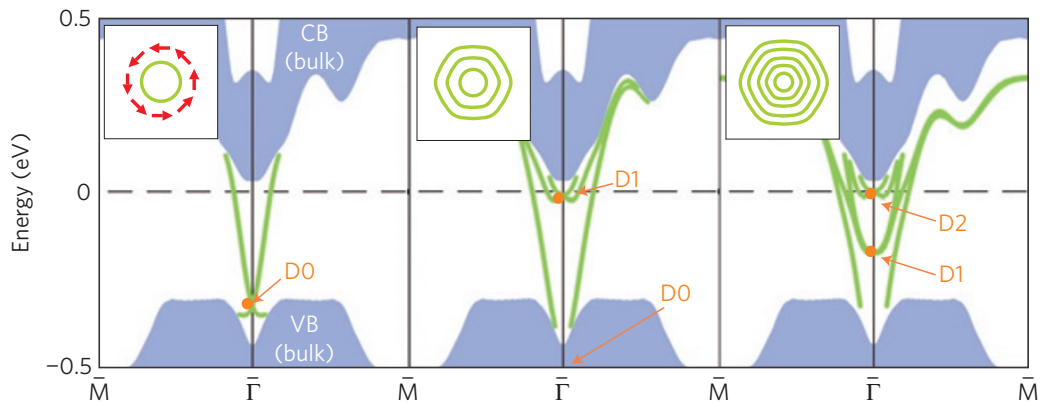

c



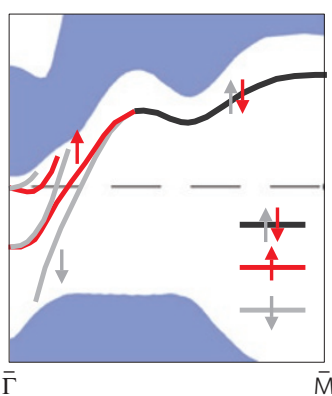

d

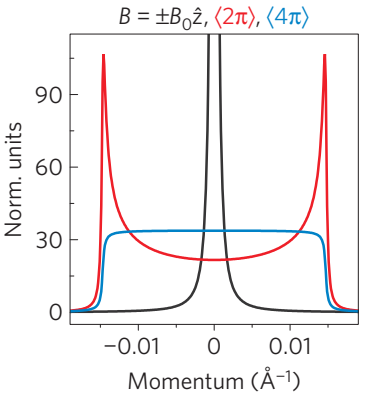

Figure 3 | Surface-state theoretical simulations. a, A first-principles GGA model of $\mathrm{Bi}_{2} \mathrm{Se}_{3}$ surface band structure (left) exhibits new Dirac points when orbital energies are lowered by $0.68 \mathrm{eV}$ on the first (centre) and the first two (right) quintuple layers of the lattice. Surface-state energy changes nonlinearly with this perturbation. Inset schematic diagrams show the Fermi-surface evolution (not to scale). b, Bands from a (right) are labelled with up arrows for right-handed and down arrows for left-handed chiral spin texture. c, The effect of Zeeman coupling on a surface-state Dirac cone defined by a Rashba Hamiltonian is considered for different magnetic-field orientations, on the basis of the surface-state Hamiltonian in ref. 12. Red arrows illustrate the path of a constant-energy ARPES measurement, corresponding to profiles in $\mathbf{d}$. $\mathbf{d}$, The domain-averaged momentum-axis distribution of a single surface-state band for a surface populated with out-of-plane ferromagnetic domains (black), in-plane domains (red) and domains with arbitrary three-dimensional orientation (blue), assuming a band velocity of $3.4 \mathrm{eVA}$ and magnetic interactions strong enough to open a $0.1 \mathrm{eV}$ gap when oriented along the $z$ axis. 

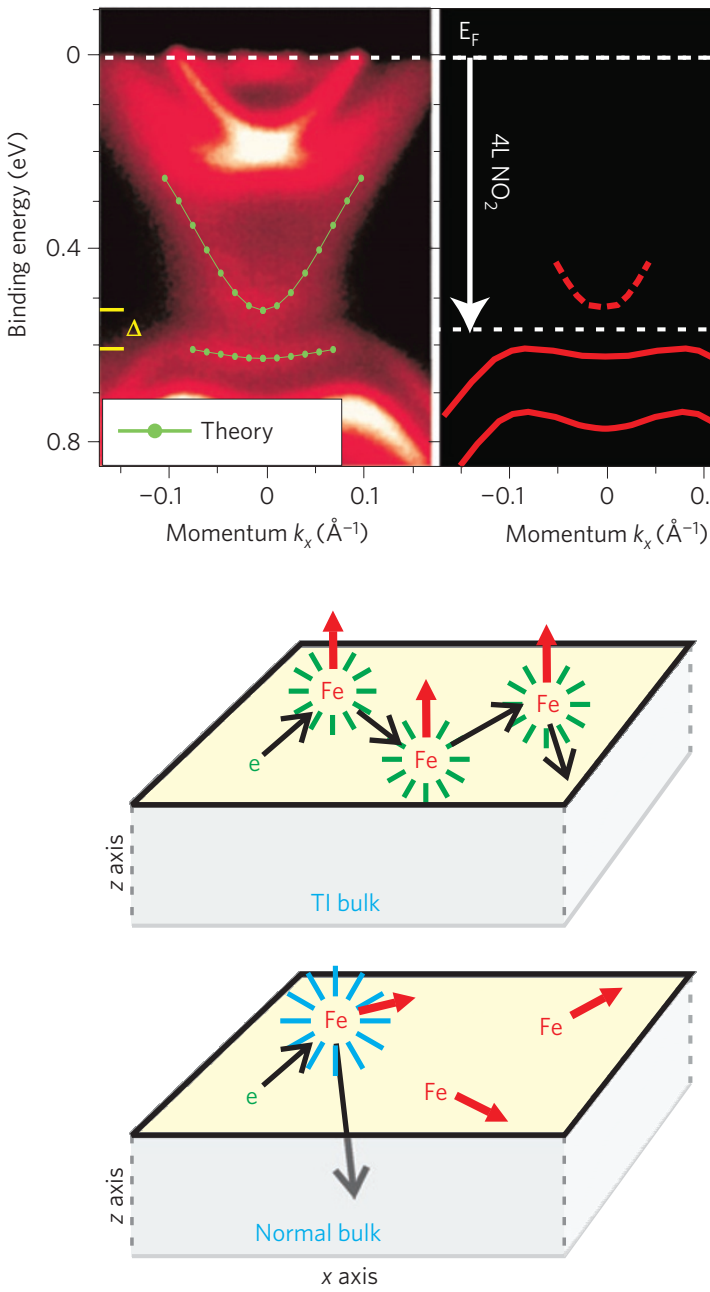



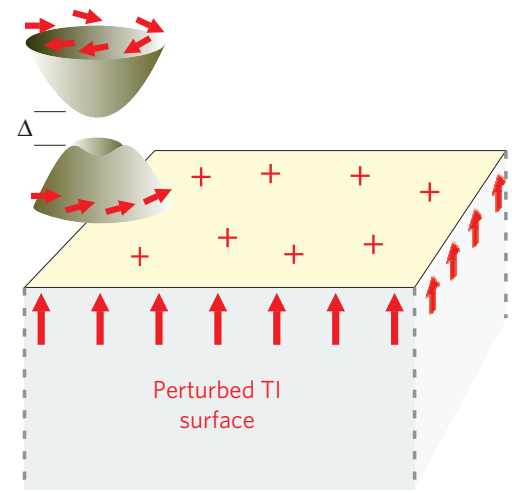

d
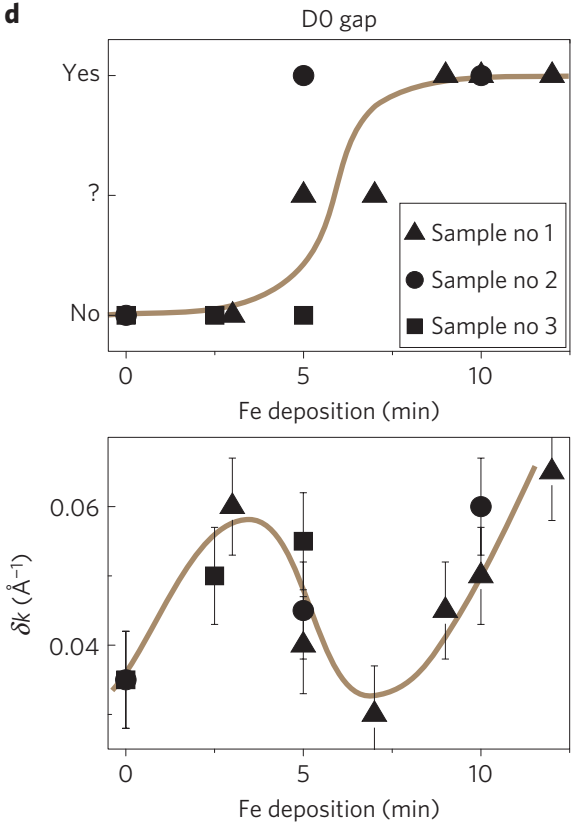

Figure 4 | Surface evolution under Coulomb interaction, disorder and magnetism. a, Left, A z-axis magnetic perturbation causes (green) GGA-predicted surface states to conform to the iron-doped dispersion. Right, A schematic diagram showing the lowering of Fermi level on the basis of molecular ( $\mathrm{NO}_{2}$ ) doping of the surface. For $\mathrm{NO}_{2}$ doping methods see ref. 6. b, A cartoon illustrates Fe deposition on the TI surface. c, Black arrows, Electron trajectories in TIs adhere to the surface, helping to mediate two-dimensional magnetic interactions. Red arrows, Fe spin. $\mathbf{d}$, The development of a gap at the lowest-energy ('DO') Dirac point (top) is compared with the half-maximum peak width ( $\delta k$ ) of connected states in the DO upper Dirac cone (bottom). Error bars represent the estimated uncertainty on the basis of the results of different fitting techniques.

from the surface environment provided by a normal semiconductor such as silicon ${ }^{11,12,26}$. When a non-magnetic crystal is doped with magnetic impurities, long-range magnetic ordering can come about as a result of itinerant electrons exchange-mediating the magnetic interaction. In a normal three-dimensional material, electrons that interact with surface-deposited magnetic impurities are free to scatter away from the surface over a $2 \pi$ solid angle, and the two-dimensional magnetic interactions therefore typically decay over several ångstroms (see, for example, ref. 27). In topological insulators, surface-state electrons are naturally confined to the surface in two-dimensional Dirac cones, and it is suggested that interactions between deposited impurities can be mediated over many nanometres (see the illustration in Fig. 4c; refs 11,26). For broader theoretical investigations and experiments with nanoscale engineering capabilities, it is interesting to note that exotic magnetic phases (for example, helical) may occur within certain specific configurations of magnetic impurities such as isolated one-dimensional chains, or from instabilities that arise as the chemical potential is moved far from the surface Dirac point ${ }^{20,24}$. In the present case of homogeneous two-dimensional deposition with chemical potential near the D0 Dirac point, the momentum-locked spin polarization of topological surface electrons is thought to support ferromagnetic order with an out-of-plane bias (B along the $\pm \hat{z}$ direction) at high impurity densities ${ }^{11,12}$, unlike direct dipole-dipole interactions, which are much weaker and favour in-plane magnetic orientation.

Our numerical simulations have shown that the new D1 and D2 Dirac-point electrons are localized deeper inside the material than D0, and are expected to interact more weakly with electronic orbitals of the surface-deposited iron. Therefore, we focus on the D0 electrons to understand the effect of iron magnetism on the topological surface. A gap appears at the D0 Dirac point after heavy iron deposition, and the lower D0 Dirac cone acquires a buckled shape with a local energy minimum at zero momentum. The mass induced in the upper D0 Dirac cone after full deposition is approximately $0.1 M_{\mathrm{e}}$ (electron masses), and the surface bandgap is about $100 \mathrm{meV}$. It is difficult to identify the exact time at which the bandgap appears, probably because there is no long-range order of 
iron spins on the surface ${ }^{28,29}$, and differently ordered domains will yield differently gapped contributions to the photoemission signal.

Global ferromagnetism breaks time-reversal symmetry, making it possible to induce a gap at the Dirac point, which is otherwise disallowed by the crystal symmetry. Figure $4 \mathrm{a}$ shows the result of modifying a generalized gradient approximation (GGA) numerical prediction of the D0 surface state by adding perturbative coupling to a surface layer of magnetic impurities with ferromagnetic out-of-plane order (equivalent to the Zeeman effect), yielding a dispersion that closely matches the experimental data. Figure $4 \mathrm{a}$ (right) is a schematic diagram showing the lowering of the Fermi level on the basis of molecular $\left(\mathrm{NO}_{2}\right)$ doping of the surface. For $\mathrm{NO}_{2}$ doping methods see ref. 6. Details of this calculation and factors that can modify the surface-state dispersion are discussed in Supplementary Information and will be an important subject for future experimental studies. If the D0 bandgap were due to an isotropic out-of-plane magnetic field, a similar gap would be expected at the D1 and D2 Dirac points, which is clearly not observed. Because magnetic-field perturbations from the submonolayer iron coating are quite weak, the magnetic-phase term in perturbative Hamiltonians can be discounted, and the dominant magnetic effects are expected to be described by a local Heisenberg-exchange term representing direct wavefunction overlap (hopping) between surface electrons and spin-polarized Fe $3 d$ orbitals $^{11}$. Evidence of Landau levels is neither seen in our data nor in this context expected from theory. The general effect of Zeeman-type magnetic symmetry breaking on topological Dirac surface electrons is shown in Fig. 3c, illustrating that out-of-plane magnetic order will cause a gap, and in-plane magnetic domains will cause bands to shift in momentum space $^{12}$. The appearance of a gap at D0 in our data is therefore consistent with a scenario in which much of the iron-deposited crystal surface is occupied by domains that have net out-ofplane magnetic moment.

Contrary to the usual trend of deposition experiments, in which photoemission images become increasingly blurry as molecules are haphazardly added to the surface, we identify a regime in which the image becomes qualitatively sharper with the increasing coverage of iron (seen in Fig. $4 \mathrm{~d}$ and the first three panels of Fig. 2a), corresponding approximately to the increasing clarity of the D0 gap. The momentum-axis width of bands $(\delta k)$ was self-consistently measured through Lorentzian fitting at binding energies between the D0 Dirac point and the onset of the D1 band structure. Doping evolution of the lower D0 Dirac cone is much less clear in the data, owing to the disordered surface and close proximity of features. Momentum width is inversely related to the electronic mean free path, and the reduction in $\delta k$ as the gap appears is probably indicative of a magnetic disorder-to-order transition, leading to a reduction in scattering. Reducing the prevalence of magnetic domains with in-plane moment could also cause the surface-state bands to seem narrower even if electronic mean free paths are unchanged, as can be observed through comparing the domain-averaged band profiles in Fig. 3d. The signature of large ferromagnetic domains with in-plane moment is a twin-peak feature that is not seen in the data. The close correspondence between reduced momentumdistribution curve or $\delta k$ width and gapping of the D0 Dirac point therefore strongly indicates the occurrence of an out-ofplane magnetic-ordering phase transition after approximately six minutes of iron deposition.

This apparent ordering transition driven by magnetic interactions that are mediated by the topological surface state is an indication of how strong topological insulator order changes the physical environment of the material surface. The observations reported here, including out-of-plane surface magnetism and the appearance of new Dirac surface states, open a window into how topological surface states are formed and interact with various perturbations. As such, they are significant for theoretical understanding of the formation of the topological insulator state in particular materials, and have direct implications for proposed devices using magnetic or Coulomb-charged interfaces with topological insulators, such as magneto-electric junctions ${ }^{12-15}$ and capacitors ${ }^{16}$ to integrate into multifunctional topological transistors.

\section{Method summary}

ARPES measurements were made at the Advanced Light Source beamlines 10 and 12 using 35.5-48 eV photons with better than $15 \mathrm{meV}$ energy resolution and overall angular resolution better than $1 \%$ of the Brillouin zone. Samples were cleaved and measured at $15 \mathrm{~K}$, in a vacuum maintained below $8 \times 10^{-11}$ torr. Momentum along the $\hat{z}$ axis is determined using an inner potential of $9.5 \mathrm{eV}$, consistent with previous photoemission investigations of undoped $\mathrm{Bi}_{2} \mathrm{Se}_{3}$ (refs 7,22). Fe atoms were deposited using an e-beam-heated evaporator at a rate of approximately $0.1 \AA \mathrm{min}^{-1}$. A quartz microbalance supplied by Leybold-Inficon with sub-ångstrom sensitivity was used to calibrate the iron-deposition flow rate. Adsorption of $\mathrm{NO}_{2}$ molecules on $\mathrm{Ca}_{x} \mathrm{Bi}_{2-x} \mathrm{Se}_{3}$ was achieved by controlled in situ exposures under static flow mode, with care to minimize photon exposure of the adsorbed surface. Large single crystals of $\mathrm{Ca}_{x} \mathrm{Bi}_{2-x} \mathrm{Se}_{3}$ were grown using methods described in the Supplementary Information. Surface- and bulk-state band calculations were carried out for comparison with the experimental data, using the linearized augmented plane-wave method implemented in the WIEN2K package ${ }^{30}$. Details of the calculation are identical to those described in ref. 7.

Received 19 June 2010; accepted 5 October 2010; published online 12 December 2010

\section{References}

1. Moore, J. E. Topological insulators: The next generation. Nature Phys. 5, 378-380 (2009).

2. Hasan, M. Z. \& Kane, C. L. Topological insulators. Rev. Mod. Phys. 82, 3045-3067 (2010).

3. Fu, L., Kane, C. L. \& Mele, E. J. Topological insulators in three dimensions. Phys. Rev. Lett. 98, 106803 (2007).

4. Hsieh, D. et al. A topological Dirac insulator in a quantum spin Hall phase. Nature 452, 970-974 (2008).

5. Hsieh, D. et al. Observation of unconventional quantum spin textures in topological insulators. Science 323, 919-922 (2009).

6. Hsieh, D. et al. A tunable topological insulator in the spin helical Dirac transport regime. Nature 460, 1101-1105 (2009).

7. Xia, Y. et al. Observation of a large-gap topological-insulator class with a single Dirac cone on the surface. Nature Phys. 5, 398-402 (2009).

8. Xia, Y. et al. Electrons on the surface of $\mathrm{Bi}_{2} \mathrm{Se}_{3}$ form a topologically-ordered two dimensional gas with a non-trivial Berry's phase. Preprint at http://arxiv.org/abs/0812.2078 (2008)

9. Hor, Y. S. et al. Development of ferromagnetism in the magnetically doped topological insulator $\mathrm{Bi}_{2-x} \mathrm{Mn}_{x} \mathrm{Te}_{3}$. Phys. Rev. B 81, 195203 (2010).

10. Hsieh, D. et al. Observation of time-reversal-protected single-Dirac-cone topological-insulator states in $\mathrm{Bi}_{2} \mathrm{Te}_{3}$ and $\mathrm{Sb}_{2} \mathrm{Te}_{3}$. Phys. Rev. Lett. 103, 146401 (2009).

11. Biswas, R. R. \& Balatsky, A. V. Impurity-induced states on the surface of 3D topological insulators. Phys. Rev. B 81, 233405 (2010).

12. Garate, I. \& Franz, M. Inverse spin-galvanic effect in a topological-insulator/ferromagnet interface. Phys. Rev. Lett. 104, 146802 (2010).

13. Fu, L. \& Kane, C. L. Probing neutral Majorana fermion edge modes with charge transport. Phys. Rev. Lett. 102, 216403 (2009).

14. Law, K. T., Lee, P. A. \& Ng, T. K. Majorana fermion induced resonant Andreev reflection. Phys. Rev. Lett. 103, 237001 (2009).

15. Qi, X-L. et al. Inducing a magnetic monopole with topological surface states Science 323, 1184-1187 (2009).

16. Seradjeh, B., Moore, J. E. \& Franz, M. Exciton condensation and charge fractionalization in a topological insulator film. Phys. Rev. Lett. 103, 066402 (2009).

17. Tse, W-K. \& MacDonald, A. H. Giant magneto-optical Kerr effect and universal Faraday effect in thin-film topological insulators. Phys. Rev. Lett. 105, 057401 (2010).

18. Zhang, H. et al. Model Hamiltonian for topological insulators. Phys. Rev. B 82, 045122 (2010).

19. Lee, D-H. Surface states of topological insulators: The Dirac fermion in curved two-dimensional spaces. Phys. Rev. Lett. 103, 196804 (2009).

20. Hasan, M. Z. et al. Warping the cone on a topological insulator. Physics 2, 108 (2009).

21. Fu, L. \& Berg, E. Odd-parity topological superconductors: Theory and application to $\mathrm{Cu}_{x} \mathrm{Bi}_{2} \mathrm{Se}_{3}$. Phys. Rev. Lett. 105, 097001 (2010). 
22. Wray, L. A., Xu, S-Y., Xia, Y., Hor, Y. S., Qian, D., Fedorov, A. V., Lin, H., Bansil, A., Cava, R. J. \& Hasan, M. Z. Observation of topological order in a superconducting doped topological insulator. Nature Phys. 6, 855-859 (2010).

23. Xia, Y. et al. Topological control: Systematic control of topological insulator Dirac fermion density on the surface of $\mathrm{Bi}_{2} \mathrm{Te}_{3}$. Preprint at http://arxiv.org/abs/0907.3089 (2009)

24. Ye, F. et al. Spin helix of magnetic impurities in two-dimensional helical metal. Europhys. Lett. 90, 47001 (2010).

25. Kawaminami, M. \& Okazaki, A. Neutron diffraction study of $\mathrm{Fe}_{7} \mathrm{Se}_{8}$. II. J. Phys. Soc. Jpn 29, 649-655 (1970).

26. Liu, Q. et al. Magnetic impurities on the surface of a topological insulator. Phys. Rev. Lett. 102, 156603 (2009).

27. Wahl, P. et al. Exchange interaction between single magnetic adatoms. Phys. Rev. Lett. 98, 056601 (2007).

28. Mermin, N. D. \& Wagner, H. Absence of ferromagnetism or antiferromagnetism in one- or two-dimensional isotropic Heisenberg models. Phys. Rev. Lett. 17, 1133-1136 (1966).

29. Zhang, R-J. \& Willis, R. F. Thickness-dependent Curie temperatures of ultrathin magnetic films: Effect of the range of spin-spin interactions. Phys. Rev. Lett. 86, 2665-2668 (2001).

30. Blaha, P. et al. Computer Code WIEN2K (Vienna Univ. Technology, 2001).

\section{Acknowledgements}

We acknowledge discussions with R. R. Biswas and D. Haldane. The synchrotron $\mathrm{X}$-ray-based measurements and theoretical computations are supported by the Basic Energy Sciences of the US DOE (DE-FG-02-05ER46200, AC03-76SF00098 and DE-FG02-07ER46352). Materials growth and characterization are supported by NSF/DMR-0819860 and NSF-DMR-1006492. M.Z.H. acknowledges extra support from the A. P. Sloan Foundation.

\section{Author contributions}

L.A.W., S-Y.X. and Y.X. contributed equally to the experiment with assistance from D.H. and M.Z.H.; A.V.F. provided beamline assistance; Y.S.H. and R.J.C. provided single-crystal samples; H.L. and A.B. carried out the calculations with assistance from M.Z.H.

\section{Additional information}

The authors declare no competing financial interests. Supplementary information accompanies this paper on www.nature.com/naturephysics. Reprints and permissions information is available online at http://npg.nature.com/reprintsandpermissions. Correspondence and requests for materials should be addressed to M.Z.H. 